\title{
Functional and Muscle-Size Effects of Flywheel Resistance Training with Eccentric-Overload in Professional Handball Players
}

\author{
by \\ Sergio Maroto-Izquierdoㅁ, David García-López², José A. de Paz
}

The aim of the study was to analyse the effects of 6 week (15 sessions) flywheel resistance training with eccentric-overload (FRTEO) on different functional and anatomical variables in professional handball players. Twentynine athletes were recruited and randomly divided into two groups. The experimental group $(E X P, n=15)$ carried out 15 sessions of FRTEO in the leg-press exercise, with 4 sets of 7 repetitions at a maximum-concentric effort. The control group (CON, $n=14$ ) performed the same number of training sessions including 4 sets of 7 maximum repetitions (7RM) using a weight-stack leg-press machine. The results which were measured included maximal dynamic strength (1RM), muscle power at different submaximal loads (PO), vertical jump height (CMJ and SJ), $20 \mathrm{~m}$ sprint time (20 m), T-test time (T-test), and Vastus-Lateralis muscle (VL) thickness. The results of the EXP group showed a substantially better improvement $(p<0.05-0.001)$ in $P O, C M J, 20 \mathrm{~m}$, T-test and $V L$, compared to the CON group. Moreover, athletes from the EXP group showed significant improvements concerning all the variables measured: $1 R M$ ( $E S=$ $0.72), P O(E S=0.42-0.83), C M J(E S=0.61), S J(E S=0.54), 20 m(E S=1.45), T$-test $(E S=1.44)$, and $V L(E S=0.63$ - 1.64). Since handball requires repeated short, explosive effort such as accelerations and decelerations during sprints with changes of direction, these results suggest that FRTEO affects functional and anatomical changes in a way which improves performance in well-trained professional handball players.

Key words: muscle power, hypertrophy, iso-inertial, sport performance.

\section{Introduction}

Muscle strength and power are critical in competitive team sports as these abilities are the basis of specific actions that determine performance (i.e. throwing, jumping, running and hitting). In these sport modalities, that include handball, players are required to repeatedly carry out short, explosive efforts such as accelerations and decelerations during sprints with changes of direction. Handball involves high-intensity short duration exercise, requiring a well-developed aerobic fitness, speed and strength (Massuca et al., 2014). Therefore, new training methods are continuously being sought to improve the ability of skeletal muscles to develop explosive strength (Perez-Gomez et al., 2013). Common exercises for explosive-strength improvement include plyometrics, ballistic exercises and weightlifting (i.e. power snatch).

As an alternative to these traditional methods, inertial-resistance training emerges as a paradigm that can be applied through different systems, such as flywheel devices. Flywheel devices provide a gravity-independent stimulus (Alkner et al., 2003; Trappe et al., 2004) that in addition to causing greater muscle activation (Caruso et al., 2006; Norrbrand et al., 2010) allows

1 - Institute of Biomedicine (IBIOMED), University of León, Spain.

2 - Department of Health Sciences, European University Miguel de Cervantes, Spain. 
for brief episodes of eccentric-overload (EO) (Berg et al., 1994; Tesch et al., 2004). Given the general consensus which exists regarding the importance of eccentric action in resistance training, induced hypertrophy (Hather et al., 1991) and strength gains (Dudley et al., 1991), the main application of flywheel-resistance training with eccentricoverload (FRTEO) is to develop muscle mass (Fernandez-Gonzalo et al., 2014a; Norrbrand et al., 2008; Seynnes et al., 2007; Tesch et al., 2004), and specially to avoid muscle atrophy caused by weightlessness situations (Alkner et al., 2003, 2004; Rittweger et al., 2007). These benefits have also been observed in people with chronic diseases that involve functional impairment (i.e. strokes and multiple sclerosis) (FernandezGonzalo et al., 2014b), and in the elderly (Brzenczek-Owczarzak et al., 2013; Hortobagyi et al., 2009).

Specifically in competitive sport, FRTEOrelated research has been focused on the prevention and treatment of injuries (Askling et al., 2003; de Hoyo et al., 2015a; Romero-Rodriguez et al., 2011) and as a part of warm-up routines (de Hoyo et al., 2014). Much less is known about the effects of FRTEO on athletic performance and muscle hypertrophy in well-trained athletes. De Hoyo and co-workers have recently reported significant improvements in vertical jump height, sprint time (10 $\mathrm{m}$ and $20 \mathrm{~m}$ ) (de Hoyo et al., 2015a) and kinetic variables during changes of direction (de Hoyo et al., 2016) after 10 week FRTEO carried out by junior soccer players. These results reinforce the input of Askling et al. (2003). In addition, Tous-Fajardo et al. (2016) suggested that iso-inertial EO in combination with vibration training performed once a week could serve as a viable adjunct to improve performance tasks specific to soccer such as changes of direction and linear speed abilities. Naczk et al. (2013, 2014, 2016) have also observed significant improvements in muscle power in healthy active men, although these benefits have not been demonstrated in athletes. To the best of our knowledge, no studies have analyzed the FRTEO chronic effects on handball players.

Therefore, the aim of this study was to analyse the effects of 6 week FRTEO on maximal dynamic strength and power output at different submaximal loads, vertical jump height, sprint time, agility and muscle thickness in professional handball players. These effects were compared to those induced by traditional in-season weight training. We hypothesized that FRTEO would lead to a higher increase in all functional, structural and dependent variables that were analysed.

\section{Methods}

A randomized controlled study was designed to compare the effects of FRTEO with those induced by traditional weight training in a group of professional handball players. Twentynine athletes completed six weeks (15 sessions) of resistance training with the leg-press exercise. Participants were randomly divided into 2 groups: an experimental group (EXP, $\mathrm{n}=15$ ), which carried out the leg press on a FRTEO basis, and a control group $(\mathrm{CON}, \mathrm{n}=14)$, which performed a similar program (volume and intensity) using a traditional weight-stack machine. Each week included 2-3 sessions consisting of 4 sets of 7 repetitions performed at maximal concentric effort. Before and after the training program, athletes came to the lab on four occasions separated by a minimum of $24 \mathrm{~h}$. The first test session was used in order to collect descriptive data and to familiarize the players from the EXP group with the flywheel leg-press device. Vastus-Lateralis muscle thickness was also measured during this first experimental session. The 1RM test was carried out during the second test session. The third session was used to measure maximal power output with different loads. The last test session was focused on measuring vertical jump height, $20 \mathrm{~m}$ sprint time and T-test time.

\section{Participants}

Twenty-nine professional male handball players were recruited from the same handball club (Club Balonmano Atlético Valladolid), which played in the first division of the Spanish handball league (ASOBAL). The biometric data and characteristics of the participants are presented in Table 1. Their regular weekly exercise practice consisted of 4 handball sessions $(\sim 9$ h), 3 sessions including strength/power exercises, 3 on-track specific physical training sessions (changes of direction and plyometric exercises) and 1 competitive match (at the weekends). The participants were highly experienced with free-weight resistance exercises, 
but none of them had previously used flywheel devices. Before giving their written informed consent to participate, athletes were informed of the purposes, benefits and risks involved in the study, being aware that they could terminate their participation in the study at any time. The study protocol was approved by the Ethics Committee of the University of León, and conformed to the standards set by the Declaration of Helsinki.

Concerning the annual periodization, the study was carried out during the beginning of the second round of the first division of the Spanish handball league (January to February). The main exclusion criteria used for this study were to have had a lower limb joint injury in the 6 months prior to the study (Fernandez-Gonzalo et al., 2014a), and/or severe lower limb muscle injury (strains for more than 27 days) in the previous 2 months (de Hoyo et al., 2014). Athletes who suffered an injury during the experimental phase of the study were excluded, as well as participants who did not follow the prescribed training program or players that did not carry out the exercises correctly. None of the participants were excluded from the study. Moreover, athletes were not allowed to change their sleeping, eating, and drinking habits throughout their participation in the study.

\section{Measures}

\section{Maximal Dynamic Strength}

The 1RM leg-press was assessed using a previously established protocol (FernandezGonzalo et al., 2014a). A $45^{\circ}$ leg press device (Ortus Fitness S.L., Valencia, Spain) was used. Briefly, after a warm-up on the leg press, participants attempted to lift a progressively increased load, with $3 \mathrm{~min}$ of rest allowed between attempts. The 1RM value was obtained using as few attempts as possible (5 attempts maximum). To consider a repetition as valid, a knee full extension $\left(180^{\circ}\right)$ had to be reached, starting from a $90^{\circ}$ knee flexion.

Power Output

The same leg press device employed in $1 \mathrm{RM}$ testing was used to measure maximal concentric power output (PO) at different intensities (50,60, 70, 80 and $90 \%$ of $1 \mathrm{RM})$. For each load, participants completed a set of 3 repetitions from $90^{\circ}$ knee flexion to full extension $\left(180^{\circ}\right)$ with a $3 \mathrm{~min}$ recovery between sets. To avoid any use of the stretch-shortening cycle, each repetition started from a complete static position, at $90^{\circ}$ knee flexion. Players were requested to perform the concentric phase of each repetition as fast as possible. Power for each repetition was sampled at $100 \mathrm{~Hz}$ using a rotary encoder (TFORCE Dynamic Measurement System, Ergotech Consulting S.L., Murcia, Spain) and the associated software (T-Force v. 2.28). The repetition with the highest mean power at each load was used for further analysis. The order of tested intensities was randomized without replacement. Participants were aware of the percentage and amount of weight they were using. For the posttraining evaluation, the same order was used as in the pre-training session.

Vertical Jump

Countermovement jump (CMJ) and squat jump (SJ) heights were assessed using a contact platform (ChronoJump, BoscoSystem, Barcelona, Spain) and the associated software (ChronoJump 1.5.0). Participants performed three attempts of each vertical jump, trying to jump as high as possible with their hands on the hips. For the SJ, the eccentric phase was eliminated, starting from a standardized position with a $90^{\circ}$ knee flexion. The CMJ started from a standing position without any pause between the eccentric and concentric phase. The knee flexion angle was self-selected. A $30 \mathrm{~s}$ active recovery was allowed between following attempts. The highest value of the SJ and CMJ was selected for further analysis.

Speed and Agility

$20 \mathrm{~m}$ sprint time and T-test time were measured using photocells (ChronoJump, BoscoSystem, Barcelona, Spain) and the associated software (ChronoJump 1.5.0). The $20 \mathrm{~m}$ sprint test consisted of a maximal intensity straight sprint between two photocell gates separated by $20 \mathrm{~m}$ from a standing position. The T-test was administered using the protocol outlined by Pauole et al. (2000) with minor modifications. The measurement of time started when players passed the electronic sensors, and stopped the instant the participants crossed the sensor plane again. Three trials were performed for each test, with a $2 \mathrm{~min}$ active recovery period between attempts. All assessments were performed on a competition parquet surface and players wore handballspecific shoes. The fastest trial was used for statistical analysis. 
Vastus Lateralis muscle thickness

The thickness of the Vastus Lateralis (VL) of the right leg of all participants was estimated using a previously described protocol (Alegre et al., 2014, 2015). VL muscle was measured in vivo by B-mode ultrasonography (MyLab ${ }^{\mathrm{TM}} 50$, Esaote, Genoa, Italy) with a $5 \mathrm{~cm}, 12 \mathrm{MHz}$ linear array probe, which was coated in water-soluble transmission gel to provide acoustic contact without depressing the dermal surface. Before measurements, the subjects were laid on a couch for $15 \mathrm{~min}$ to allow osmotic fluids to shift. During measurements, the participants were laid in a supine position with their knees fully extended and muscles relaxed. The images for the measurements of muscle thickness were recorded at 25,50 and $75 \%$ distance between the greater trochanter and lateral condyle of the femur (VL25, VL50 and VL75, respectively), with the ultrasound probe placed on the transversal plane and perpendicular to the skin. VL muscle thickness was determined by measuring the perpendicular distance between the medial and lateral limits of the muscle with ultrasound software $\left(\mathrm{MyLab}^{\mathrm{TM}}\right.$ Desk, Esaote, Genoa, Italy) before and after the training intervention. In order to increase the reliability of the obtained data, the measuring points were marked daily on the skin with indelible ink and muscle architecture images from before and after the intervention were compared.

\section{Procedures}

Participants performed a lower-limb resistance-training program based on the leg press exercise. The training program lasted 6 weeks, and included 2 (weeks 1, 3, 5) or 3 (weeks 2, 4, 6) sessions per week, with at least 48 hours between sessions (Fernandez-Gonzalo et al., 2014a; Norrbrand et al., 2008). Each session included 4 sets of 7 repetitions, with a 3 min recovery period between sets. Each training session started with a warm-up consisting of 5 min cycling and 2 sets of 10 non-maximal repetitions in specific leg press exercises. The flywheel device (Leg Press, Inc YoYo Technology, Stockholm, Sweden) used by the EXP group was equipped with two $6.5 \mathrm{~kg}$ flywheels $(45 \mathrm{~cm}$ diameter) with moment inertia of $0.145 \mathrm{~kg} \cdot \mathrm{m}^{2}$ (Figure 1).

FRTEO performed by the EXP group consisted of accelerating/braking flywheels through the extension-flexion of the knees and hips, as previously described (Fernandez-Gonzalo et al., 2014a). Briefly, each repetition consisted of a maximum concentric action of thrust on the platform where the feet were placed, rotating the wheels. Participants were asked to push with maximal effort through the entire concentric action, which ranged from $70^{\circ}$ knee flexion to near full extension. At the end of this concentric action, the flywheel strap wound back due to inertial forces, which initiated the reversed eccentric action. During the first third of the eccentric action, participants were instructed to resist gently, and thereafter, to apply maximal breaking force to stop the movement at about $70^{\circ}$ knee flexion (Fernandez-Gonzalo et al., 2014a). By means of this approach, EO was produced (Norrbrand et al., 2010; Romero-Rodriguez et al., 2011; Tesch et al., 2004).

The CON group carried out leg presses on a traditional weight-stack machine (Ortus Fitness S.L., Valencia, Spain). They performed the same training volume with a load equivalent to 7 repetitions maximum (7RM) for each set. Participants were instructed to perform the concentric phase of each repetition at maximal velocity. At the end of each set, the participants were asked to rate their perceived exertion according to a resistance-training specific scale (OMNI scale) (Lagally et al., 2006). The athletes were accustomed to the OMNI scale, as strength and conditioning staff used it regularly during pre-season and in-season workouts. Research personnel provided strong verbal encouragement during all repetitions performed by players from both groups.

\section{Statistical Analysis}

Statistical analysis was performed with SPSS v.20.0 (SPSS Inc., IBM, USA). The data is presented as mean \pm SEM. Data distribution was checked for normality using the Shapiro-Wilk test. VL thickness, 1RM, power at different loads, SJ, CMJ, $20 \mathrm{~m}$ sprint time and T-test time were examined using a two-way ANOVA. The 2 factors considered were experimental condition (EXP vs. $\mathrm{CON}$ ) and time (PRE vs. POST). When a significant interaction was found, post hoc comparisons with Bonferroni adjustment were employed. The effect size (ES) of training was calculated for paired variables (Cohen, 1998). Cohen suggested ES of $0.2,0.5,0.8$ and 1.3 to represent small, moderate, large and very large effects, respectively. Practical relevance was 
defined as an ES $>0.8$ (Hopkins et al., 2009). The significance level was set at $p<0.05$.

\section{Results}

Participants from both groups completed all the designed training sessions. Perceived exertion reported after the training sets was similar in the EXP and CON groups. Table 2 displays maximal dynamic strength (1RM), power output, vertical jump, sprint time and agility results.

\section{Maximal Dynamic Strength}

A significant time effect was observed $\left(\mathrm{F}_{1,29}=33.71\right)$. Thus, the EXP $(12.2 \%, p<0.001$; ES $=$ $0.72)$ and $\mathrm{CON}(7.9 \%, p<0.01$; ES $=0.49)$ groups showed a significant increase in $1 \mathrm{RM}$ value. No significant condition or condition $\mathrm{x}$ time main effects were observed.

Power Output

Regarding $\mathrm{PO}$, there was a significant time effect for all the loads tested: PO50 $\left(\mathrm{F}_{1,29}=\right.$ 10.247; $p<0.01)$, PO60 ( $\left.\mathrm{F}_{1,29}=7.576 ; p<0.05\right), \mathrm{PO} 70$ $\left(\mathrm{F}_{1,29}=13.344 ; p<0.01\right), \operatorname{PO} 80\left(\mathrm{~F}_{1,29}=10.454 ; p<\right.$ $0.01)$ and PO90 $\left(\mathrm{F}_{1,29}=5.195 ; p<0.05\right)$. Although no significant condition or condition $\mathrm{x}$ time main effects were observed, only the EXP players showed significant changes in $\mathrm{PO}$, with increases ranging from 10 to $21.6 \%$ ( $E S=0.42-0.83$ ).

Vertical Jump

A significant time effect was observed in SJ $\left(\mathrm{F}_{1,29}=26.233 ; p<0.001\right)$, with significant increases showed by the CON $(p<0.05, \mathrm{ES}=0.36)$ and $\operatorname{EXP}(p<0.001 ; \mathrm{ES}=0.54)$ groups. No significant condition or condition $\mathrm{x}$ time main effects were observed.

Results regarding the $\mathrm{CMJ}$ indicated a significant time $\left(\mathrm{F}_{1,29}=28.452 ; p<0.001\right)$, condition $\left(\mathrm{F}_{1,29}=5.513 ; p<0.05\right)$ and condition $\mathrm{x}$ time $\left(\mathrm{F}_{1,29}=\right.$ 5.691; $p<0.05)$ main effects. Thus, only the EXP participants enhanced their $\mathrm{CMJ}$ value after the training period $(p<0.001$; ES $=0.61)$. Post-hoc analysis pointed out that the $\mathrm{CMJ}$ value after the training period was significantly higher in the EXP group in comparison to the CON group $(p<$ 0.05).

Speed and Agility

Significant time $\left(\mathrm{F}_{1,29}=58.417 ; p<0.001\right)$ and condition $\mathrm{x}$ time $\left(\mathrm{F}_{1,29}=6.503 ; p<0.05\right)$ main effects were observed concerning the $20 \mathrm{~m}$ sprint time. Post-hoc analysis revealed that both groups significantly reduced their sprint time (EXP: $p<$ $0.01, \mathrm{ES}=1.45 ; \mathrm{CON}: p<0.01, \mathrm{ES}=0.85$ ), with a larger reduction in the EXP group.

Results regarding T-test time showed significant time $\left(\mathrm{F}_{1,29}=26.345 ; p<0.001\right)$, condition $\left(\mathrm{F}_{1,29}=5.226 ; p<0.05\right)$ and condition $\mathrm{x}$ time $\left(\mathrm{F}_{1,29}=\right.$ 5.713; $p<0.05)$ main effects. Thus, only the EXP participants significantly reduced their T-test time value after the training period $(p<0.001$; ES $=$ 1.44). Moreover, post-hoc analysis pointed out that the T-test time after the training period was significantly higher in the EXP group in comparison to the CON group $(p<0.05)$.

Vastus-Lateralis muscle thickness

Results regarding Vastus-Lateralis muscle thickness are displayed in Figure 2.

A significant time effect was observed concerning VL25 $\left(\mathrm{F}_{1,29}=22.801 ; p<0.001\right)$, VL50 $\left(\mathrm{F}_{1,29}=168.772 ; p<0.001\right)$ and VL75 $\left(\mathrm{F}_{1,29}=39.229 ; p\right.$ $<0.001)$. Likewise, data showed a significant interaction condition $\mathrm{x}$ time effect for VL25 $\left(\mathrm{F}_{1,29}=\right.$ $15.028 ; p<0.01)$, VL50 $\left(\mathrm{F}_{1,29}=74.665 ; p<0.001\right)$ and VL75 $\left(\mathrm{F}_{1,29}=9.651 ; p<0.01\right)$. Thus, the EXP participants showed a significant VL hypertrophy at proximal $(p<0.001$; ES = 1.61), medial $(p<0.001$; $\mathrm{ES}=1.64)$ and distal $(p<0.001 ; \mathrm{ES}=0.63)$ levels. Although athletes from the $\mathrm{CON}$ group also showed a significant increase in muscle thickness at media (VL50, $p<0.05$; ES $=0.39$ ) and distal $(V L 75, p<0.05$; ES = 0.15) measurements, the VL muscle hypertrophy experienced by the EXP participants was significantly higher $(p<0.001$ 0.01 ) than that showed by the CON group at all measurement levels (VL25, VL50 and VL75).

\begin{tabular}{|c|c|c|c|c|}
\hline & & & & Table 1 \\
\hline \multicolumn{5}{|c|}{ Descriptive data of the participants, Mean \pm SEM } \\
\hline & Age (y) & $\begin{array}{l}\text { Body height } \\
(\mathrm{cm})\end{array}$ & $\begin{array}{l}\text { Body mass } \\
(\mathrm{kg})\end{array}$ & $\begin{array}{l}\text { Body mass } \\
\text { index }\left(\mathrm{kg} / \mathrm{m}^{2}\right)\end{array}$ \\
\hline Experimental Group & $19.8 \pm 1$ & $186 \pm 8$ & $82.3 \pm 3.3$ & $23.7 \pm 0.6$ \\
\hline Control Group & $23.8 \pm 1.6$ & $184 \pm 1$ & $85.6 \pm 3.7$ & $25.2 \pm 0.9$ \\
\hline
\end{tabular}


Table 2

1RM, PO at different loads, SJ, CMJ, 20 m and T-test results before and after the training period for the EXP and CON groups. Values are mean \pm SEM

\begin{tabular}{lcccccc}
\hline \multicolumn{4}{c}{ EXPERIMENTAL } & \multicolumn{3}{c}{ CONTROL } \\
\hline Pre & Post & $\Delta \%$ & Pre & Post & $\Delta \%$ \\
\hline PO50 (w) $(\mathrm{w})$ & $258.7 \pm 9.5$ & $290.3 \pm 12.5$ & 12.2 & $242.9 \pm$ & $262 \pm 9.1 *$ & 7.9 \\
& $835.1 \pm 30.4$ & $943.1 \pm 59^{* *}$ & 12.9 & $894.2 \pm$ & $943.4 \pm 47.4$ & 5.5 \\
PO60 (w) & $918 \pm 39.2$ & $1009.5 \pm 67.4$ & 10 & $908.3 \pm$ & $954.9 \pm 45.1$ & 5.1 \\
& & $1030.4 \pm 65.4$ & 12.4 & $922.5 \pm$ & $982.7 \pm 37.6$ & 6.5 \\
PO70 (w) & $916.8 \pm 38.6$ & & & & \\
PO80 (w) & $840.2 \pm 38.7$ & $1005.8 \pm 68.7$ & 19.7 & $886.2 \pm$ & $948.4 \pm 34.8$ & 7 \\
PO90 (w) & $777.4 \pm 42$ & $944.9 \pm 70^{*}$ & 21.6 & $871.9 \pm 41$ & $941 \pm 42.7$ & 7.9 \\
SJ (cm) & $33.4 \pm 1.5$ & $36.7 \pm 1.5 * * *$ & 9.6 & $31.5 \pm 1.2$ & $32.9 \pm 0.8 *$ & 4.5 \\
CMJ (cm) & $35.7 \pm 1.3$ & $39.2 \pm 1.5^{* * *}$ & 9.8 & $33 \pm 1.2$ & $34.1 \pm 0.9$ & 3.4 \\
20m (s) & $3.7 \pm 0.1$ & $3.3 \pm 0.1^{* * *}$ & -10 & $3.6 \pm 0.1$ & $3.5 \pm 0.1 * *$ & -5.1 \\
T-test (s) & $9.2 \pm 0.1$ & $8.6 \pm 0.1^{* * *}$ & -7 & $9.5 \pm 0.2$ & $9.1 \pm 0.1$ & -4.4 \\
\hline
\end{tabular}

Abbreviations: 1RM, maximal dynamic strength; $P O$, power output;

PO50-90 maximal concentric power output (PO) at different intensities

(50, 60, 70, 80 and $90 \%$ of $1 R M)$; SJ, squat jump;

CMJ, countermovement jump; $20 \mathrm{~m}, 20 \mathrm{~m}$ sprint time.

* Significantly different from pre-training value, where ${ }^{*} p<0.05,{ }^{* *} p<0.01$, and ${ }^{* * *} p<0.001$.

$\$$ Significantly different from CON group value, where $\$ p<0.05$ and $\$ \$ p<0.01$.

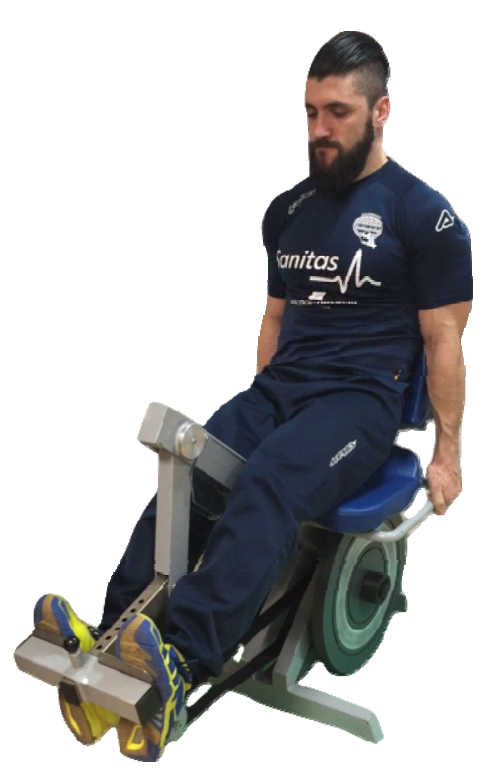

Figure 1

Iso-inertial device used in the FRTEO program. 


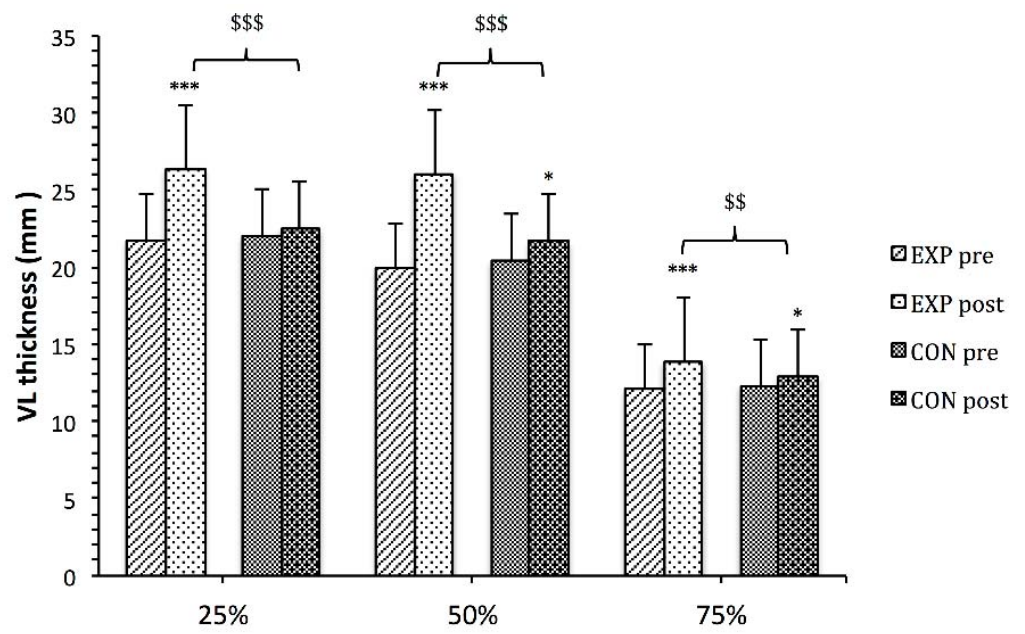

Figure 2

Vastus Lateralis thickness at proximal (25\%), medial (50\%) and distal (75\%) measurements before and after the training program for both, experimental (EXP) and control (CON) groups. * Significantly different from pre-training value, where

${ }^{*} p<0.05$ and ${ }^{* * *} p<0.001$. \$ Significantly different from CON group value, where $_{p}<0.05$ and $\$ \$ p<0.001$.

\section{Discussion}

In addition to technical and tactical game skills, performance in handball is related to power, strength, speed and agility (Massuca et al., 2014). Therefore, training approaches that lead to improvements in those abilities should be taken into account by coaches as well as strength and conditioning specialists focused on handball performance. To the best of our knowledge, this is the first study comparing functional and structural effects of a flywheel resistance-training program with eccentric-overload (FRTEO) to those induced by a traditional weight-training program, in professional handball players. The main finding pointed out by the results is that 6 week FRTEO including 15 sessions with 4 sets of 7 repetitions performed at maximal concentric intensity using the leg press exercise induced significant improvements in maximal dynamic strength (1RM), maximal power at different loads, vertical jump height, $20 \mathrm{~m}$ sprint time and T-test time. Moreover, FRTEO induced significant hypertrophy in Vastus-Lateralis muscle at proximal (VL25), medium (VL50) and distal (VL75) levels. All these positive effects were significantly higher than those showed by subjects who carried out a similar (volume and perceived effort) training program on a conventional leg/press weight/stack machine.

During traditional concentric-eccentric resistance exercise performed at maximal intensity, the eccentric phase is clearly underloaded (e.g. about 40-50\%) which has been shown in several studies (Dudley et al., 1991; Hather et al., 1991). These studies used a variety of experimental manipulation and demonstrated the role of eccentric contractions to improve contractile characteristics and muscle size in humans. It makes sense to apply eccentricoverloads during resistance training in order to increase neuromuscular adaptations (Hortobagyi 
et al., 2001). Specific literature includes research studies in which this EO is applied through an iso-inertial gravity-independent stimulus, with positive results concerning structural (Alkner et al., 2003, 2004; Tesch et al., 2004) and functional adaptations (de Hoyo et al., 2015a; FernandezGonzalo et al., 2014a; Romero-Rodriguez et al., 2011) when applied to different populations, mainly recreationally trained subjects or physical education students. Our results point out that applying FRTEO can significantly increase the 1RM value in well-trained handball players, although this improvement is similar to that obtained after a traditional weight training program. Although the participants of the EXP group were exposed to EO, the $1 \mathrm{RM}$ test carried out was similar in nature to training performed by the CON group. After a similar 6 week FRTEO study (Fernandez-Gonzalo et al., 2014a) which was also carried out with the leg press exercise, a group of physical education students increased the $1 \mathrm{RM}$ value by $25 \%$. As the participants in our study were professional handball players and thus well-trained athletes, the lower increase observed in maximal dynamic strength of the athletes compared to the students could be due to the initial physical ability of the athletes before the study.

The significant improvements concerning $\mathrm{PO}$ at different intensities (50, 60, 70, 80 and 90\% of 1RM) after FRTEO carried out by the EXP participants are in line with previous studies. Moreover, Fernandez-Gonzalo and co-workers ( 2014a) also observed significant increases in muscle power at loads equivalent to 60,70 and $80 \%$ of the $1 \mathrm{RM}$ after similar FRTEO. Furthermore, after analysing a sample of students, Naczk et al. (2013, 2014, 2016) showed significant muscle power improvements in lower and upper limbs. However, our study is the first to demonstrate the positive effects of FRTEO on increasing muscle power at high percentages of $1 \mathrm{RM}$ in athletes. Interestingly, participants from the CON group did not increase their PO at any load, even though the training stimulus could be considered more similar in nature to that found during the PO test. These major improvements in muscle power may be explained by the particular characteristics of flywheel training to induce EO, since the eccentric EO is usually applied in the last portion of the range of motion during the eccentric action. That is, EO is produced mainly at a joint angle close to $70^{\circ}$ during the leg press just before the subsequent concentric action is initiated. This exercise technique would maximize the stretch shortening cycle, allowing for greater production of force during the first part of the concentric action, and therefore, a higher velocity during the entire movement (i.e. increased power).

PO results together with vertical jump (SJ and $\mathrm{CMJ}$ ) and significant improvements observed in the EXP group indicate that FRTEO appears to be an effective tool to increase muscle power in well-trained handball players. Although a similar training program carried out by physical education students did not induce any significant increases in SJ or CMJ (Fernandez-Gonzalo et al., 2014a), a study focused on elite soccer players indicated significant gains in CMJ height after 10week FRTEO (de Hoyo et al., 2015a). The significant interaction condition $\mathrm{x}$ time showed by our results in regard to $\mathrm{CMJ}$ height could suggest that vertical jump improvements induced by FRTEO are higher in comparison with those induced by traditional resistance training when the jump modality includes an eccentric phase (i.e. CMJ). Since leg power has been suggested as an essential component for elite handball players (Massuca et al., 2014), these results could support the inclusion of FRTEO in handball training routines. In a recent study, de Hoyo et al. (2015b) showed that under specific circumstances, i.e. FW exercise performed in the horizontal plane vs. free weights using a guided (vertical) squat exercise, the gains in jumping height were lower for FW compared to traditional training.

As quickness and agility are characteristics of elite handball players (Massuca et al., 2014), the present results concerning $20 \mathrm{~m}$ sprints and T-tests indicate that FRTEO is a useful training approach producing performance-related adaptations. These adaptations are relevant given the importance of the decelerating phases in the sprinting technique, either in a linear or in a change-of-direction approach. Efficient eccentric activation timing and force production of the quadriceps and hamstring muscle groups are necessary during the stance phase in order to provide better elastic energy absorption and storage for impulse during take-off. In the same line, De Hoyo and co-workers (2015a, 2016) 
showed significant improvements in kinetic variables, changes of direction and $20 \mathrm{~m}$ sprints time after 10 week FRTEO carried out with half squat and leg curl flywheel devices by junior elite soccer players. Similar to linear-sprint results, and probably more representative in terms of performance, our data pointed out a significant improvement in the change-of-direction test, such as the T-test. These results are in line with TousFajardo et al. (2016) who suggested that flywheel training performed once a week could serve as a viable adjunct to improve performance tasks specific to soccer.

Greater muscle mass is often an advantageous characteristic in sports, as in team handball, where speed and explosiveness are the essence of the sport (Massuca et al., 2014). Our results indicate that the significant increase in Vastus-Lateralis muscle thickness achieved after the FRTEO program was significantly higher than that induced by a traditional weight training program. The efficacy of FRTEO to induce muscle hypertrophy has been well documented (Fernandez-Gonzalo et al., 2014a; Norrbrand et al., 2008; Tesch et al., 2004). After a similar FRTEO training program, physical education students also showed significant hypertrophy of the thigh muscles (Fernandez-Gonzalo et al., 2014a). In a similar line, Norrbrand et al. (2008) observed a significant increase in quadriceps muscle volume after 5 week FRTEO carried out by untrained men in leg-extension exercises. At a physiological level, the enhanced eccentric load apparently led to a subtly faster gene expression pattern, inducing a faster muscle phenotype plus associated adaptations that make a muscle better suited for fast, explosive movements (Friedmann-Bette et al., 2010). However, since the mechanism of hypertrophy is not yet completely understood, future studies should delve into these potential pathways, helping us to understand the underlying mechanism of the high hypertrophic role of FRTEO.

In summary, 6-week FRTEO with 2-3 sessions a week, including 4 sets of 7 repetitions of the leg-press exercise performed at maximum concentric intensity induces significant improvements in maximal dynamic strength, power output at different loads, vertical jump height, sprint time, agility and muscle thickness in professional handball players. The adaptations regarding muscle power at different submaximal loads, countermovement jump, $20 \mathrm{~m}$ sprint time, T-test time and muscle thickness of the Vastus Lateralis were significantly larger than those observed after a similar (volume and intensity) resistance training program carried out with a traditional weight-stack leg press machine and including a similar volume and perceived effort. Therefore, the EO applied through a flywheel device seems to be a useful tool inducing functional and anatomical adaptations related with performance in well-trained handball players. Future studies should analyze FRTEO programs using different training volumes and exercises in order to reinforce these results.

\section{Acknowledgements}

Sergio Maroto-Izquierdo is supported by the Ministry of Education of Spain (FPU014/05732). The authors declare they have no competing interests.

\section{References}

Alegre LM, Aguado X, Rojas-Martin D, Martin-Garcia M, Ara I, Csapo R. Load-controlled moderate and high-intensity resistance training programs provoke similar strength gains in young women. Muscle Nerve, 2015; 51(1): 92-101

Alegre LM, Ferri-Morales A, Rodriguez-Casares R, Aguado X. Effects of isometric training on the knee extensor moment-angle relationship and vastus lateralis muscle architecture. Eur J Appl Physiol, 2014; 114(11): 2437-2446

Alkner B, Tesch PA. Efficacy of a gravity-independent resistance exercise device as a countermeasure to muscle atrophy during 29-day bed rest. Acta Physiol Scand, 2004; 181: 345-357

Alkner BA, Berg HE, Kozlovskaya I, Sayenko D, Tesch P. Effects of strength training, using a gravityindependent exercise system, performed during 110 days of simulated space station confinement. Eur J Appl Physiol, 2003; 90: 44-49 
Askling C, Karlsson J, Thorstensson A. Hamstring injury occurrence in elite soccer players after preseason strength training with eccentric overload. Scand J Med Sci Sports, 2003; 13(4): 244-250

Berg HE, Tesch A. A gravity-independent ergometer to be used for resistance training in space. Aviat Space Environ Med, 1994; 65(8): 752-756

Brzenczek-Owczarzak W, Naczk M, Arlet J, Forjasz J, Jedrzejczak T, Adach Z. Estimation of the efficacy of inertial training in older women. J Aging Phys Act, 2013; 21(4): 433-443

Caruso JF, Hernandez DA, Porter A, Schweikert T, Saito K, Cho M, De Garmo N, Nelson NM. Integrated electromyography and performance outcomes to inertial resistance exercise. J Strength Cond Res, 2006; 20(1): 151-156

Cohen J. Statistical Power Analysis for the Behavioral Sciences. (2nd ed.). Hillsdale, NJ: L.: Erlbaum Associates; 1998

de Hoyo M, de la Torre A, Pradas F, Sanudo B, Carrasco L, Mateo-Cortes J, Dominguez-Cobo S, Fernandes $\mathrm{O}$, Gonzalo-Skok O. Effects of eccentric overload bout on change of direction and performance in soccer players. Int J Sports Med, 2014; 36(4): 308-314

de Hoyo M, Pozzo M, Sanudo B, Carrasco L, Gonzalo-Skok O, Dominguez-Cobo S, Moran-Camacho E. Effects of a 10-week in-season eccentric-overload training program on muscle-injury prevention and performance in junior elite soccer players. Int J Sports Physiol Perform, 2015a; 10(1): 46-52

de Hoyo M, Sanudo B, Carrasco L, Dominguez-Cobo S, Mateo-Cortes J, Cadenas-Sanchez MM, Nimphius S. Effects of traditional versus horizontal inertial flywheel power training on common sport-related tasks. J Hum Kinet, 2015b; 47: 155-167

de Hoyo M, Sanudo B, Carrasco L, Mateo-Cortes J, Dominguez-Cobo S, Fernandes O, Del Ojo JJ, GonzaloSkok O. Effects of 10-week eccentric overload training on kinetic parameters during change of direction in football players. J Sports Sci, 2016; 34(14): 1380-1387

Dudley GA, Tesch PA, Miller BJ, Buchanan P. Importance of eccentric actions in performance adaptations to resistance training. Aviat Space Environ Med, 1991; 62(6): 543-550

Fernandez-Gonzalo R, Lundberg TR, Alvarez-Alvarez L, de Paz JA. Muscle damage responses and adaptations to eccentric-overload resistance exercise in men and women. Eur J Appl Physiol, 2014a; 114(5): 1075-1084

Fernandez-Gonzalo R, Nissemark C, Aslund B, Tesch PA, Sojka P. Chronic stroke patients show early and robust improvements in muscle and functional performance in response to eccentric-overload flywheel resistance training: a pilot study. J Neuroeng Rehabil, 2014b; 11: 150

Friedmann-Bette B, Bauer T, Kinscherf R, Vorwald S, Klute K, Bischoff D, Muller H, Weber MA, Metz J, Kauczor HU, Bartsch P, Billeter R. Effects of strength training with eccentric overload on muscle adaptation in male athletes. Eur J Appl Physiol, 2010; 108(4): 821-836

Hather BM, Tesch PA, Buchanan P, Dudley GA. Influence of eccentric actions on skeletal muscle adaptations to resistance training. Acta Physiol Scand, 1991; 143(2): 177-185

Hopkins WG, Marshall SW, Batterham AM, Hanin J. Progressive statistics for studies in sports medicine and exercise science. Med Sci Sports Exerc, 2009; 41(1): 3-13

Hortobagyi T, DeVita P, LaStayo P. Neuromuscular and balance responses to flywheel inertial versus weight training in older persons. J Biomech, 2009; 42(7): 956; author reply 957

Hortobagyi T, Devita P, Money J, Barrier J. Effects of standard and eccentric overload strength training in young women. Med Sci Sports Exerc, 2001; 33(7): 1206-1212

Lagally KM, Robertson RJ. Construct validity of the OMNI resistance exercise scale. J Strength Cond Res, 2006; 20(2): 252-256

Massuca LM, Fragoso I, Teles J. Attributes of top elite team-handball players. J Strength Cond Res, 2014; 28(1): 178-186

Naczk M, Brzenczek-Owczarzak W, Arlet J, Naczk A, Adach Z. Training effectiveness of the inertial training and measurement system. J Hum Kinet, 2014; 44: 19-28 
Naczk M, Naczk A, Brzenczek-Owczarzak W, Arlet J, Adach Z. Impact of inertial training on strength and power performance in young active men. J Strength Cond Res, 2013; [Epub ahead of print]

Naczk M, Naczk A, Brzenczek-Owczarzak W, Arlet J, Adach Z. Efficacy of inertial training in elbow joint muscles: influence of different movement velocities. J Sports Med Phys Fitness, 2016; 56(3): 223-231

Norrbrand L, Fluckey JD, Pozzo M, Tesch PA. Resistance training using eccentric overload induces early adaptations in skeletal muscle size. Eur J Appl Physiol, 2008; 102(3): 271-281

Norrbrand L, Pozzo M, Tesch PA. Flywheel resistance training calls for greater eccentric muscle activation than weight training. Eur J Appl Physiol, 2010; 110(5): 997-1005

Pauole K, Madole K, Garhammer J, Lacourse M, Rozenek R. Reliability and Validity of the T-Test as a Measure of Agility, Leg Power, and Leg Speed in College-Aged Men and Women. J Strength Cond Res, 2000; 14(4): 443-450

Perez-Gomez J, Calbet JA. Training methods to improve vertical jump performance. J Sports Med Phys Fitness, 2013; 53(4): 339-357

Rittweger J, Felsenberg D, Maganaris C, Ferretti JL. Vertical jump performance after 90 days bed rest with and without flywheel resistive exercise, including a 180 days follow-up. Eur J Appl Physiol, 2007; 100(4): 427-436

Romero-Rodriguez D, Gual G, Tesch PA. Efficacy of an inertial resistance training paradigm in the treatment of patellar tendinopathy in athletes: a case-series study. Phys Ther Sport, 2011; 12(1): 43-48

Seynnes OR, de Boer M, Narici MV. Early skeletal muscle hypertrophy and architectural changes in response to high-intensity resistance training. J Appl Physiol, 2007; 102(1): 368-373

Tesch PA, Ekberg A, Lindquist DM, Trieschmann JT. Muscle hypertrophy following 5-week resistance training using a non-gravity-dependent exercise system. Acta Physiol Scand, 2004; 180: 89-98

Tesch PA, Ekberg A, Lindquist DM, Trieschmann JT. Muscle hypertrophy following 5-week resistance training using a non-gravity-dependent exercise system. Acta Physiol Scand, 2004; 180(1): 89-98

Tous-Fajardo J, Gonzalo-Skok O, Arjol-Serrano JL, Tesch P. Enhancing Change-of-Direction Speed in Soccer Players by Functional Inertial Eccentric Overload and Vibration Training. Int J Sports Physiol Perform, 2016; 11(1): 66-73

Trappe S, Trappe T, Gallagher P, Harber M, Alkner B, Tesch P. Human single muscle fibre function with 84 day bed-rest and resistance exercise. J Physiol, 2004; 557(Pt 2): 501-513

\section{Corresponding author:}

\section{Sergio Maroto-Izquierdo}

Institute of Biomedicine (IBIOMED)

University of León, Spain

Phone: (+34) 987293019 / Fax: (+34) 987293019

E-mail: smaroi@unileon.es 